AIAA-99-2443

The Origins of Accelerator Grid Current: Analysis of T5-Grid Test Results

Mark W. Crofton

The Aerospace Corporation

El Segundo, CA

lain D. Boyd

University of Michigan

Ann Arbor, MI

35th AIAA/ASME/SAE/ASEE Joint Propulsion

Conference and Exhibit

20-24 June 1999

Los Angeles, California

For permission to copy or to republish, contact the American Institute of Aeronautics and Astronautics, 1801 Alexander Bell Drive, Suite 500, Reston, VA, 20191-4344. 


\title{
THE ORIGINS OF ACCELERATOR GRID CURRENT: ANALYSIS OF T5-GRID TEST RESULTS
}

\author{
Mark W. Crofton \\ Technology Operations, Mechanics \& Materials Technology Center \\ The Aerospace Corporation, P.O. Box 92957 \\ Los Angeles, California 90009 \\ Iain D. Boyd \\ Department of Aerospace Engineering, University of Michigan \\ Ann Arbor, Michigan 48109-2140
}

\begin{abstract}
Grid current levels were obtained at various operating points of a T5 ion thruster using flightmodel extraction grids. Because of the improved grid design and precision fabrication, better analysis of the dependence of grid current on operating point could be performed. The decel current can be explained largely in terms of impingement by charge exchange ions. However, accel current levels are found to have a major component which is independent of charge exchange and rises sharply with beam current. A DSMC-PIC model was constructed which includes the effects of momentum transfer, ion-neutral charge exchange, Coulomb collisions, and double-charge ions. Using existing far-field data on the energy distribution of beam ions to $\mathrm{fix}$ the ion temperature, excellent relative and absolute agreement between theory and experiment has been achieved. The results suggest that ion temperature in the discharge plasma can be far higher than other ion engine modeling studies have assumed. about $4 \mathrm{eV}$ in the present case. Ions with high radial velocity have relatively high probability' of impinging on the accel grid. and will do so with a higher sputter yield than the average charge exchange ion.
\end{abstract}

\section{Nomenclature}

$\begin{array}{ll}\text { A } & \text { cross sectional area } \\ \text { C } & \text { mean molecular speed } \\ \mathrm{e} & \text { elementary unit of charge } \\ \mathrm{E} & \text { kinetic energy } \\ \mathrm{g} & \text { relative velocity } \\ \mathrm{I}_{\text {beam }} & \text { beam current }\end{array}$

$\begin{array}{ll}\mathrm{I}_{\text {acc }} & \text { total accel current } \\ \mathrm{I}_{\text {acc }}^{\mathrm{Ec}} & \text { accel current due to charge exchange } \\ \mathrm{I}_{\text {dec }} & \text { decel current } \\ \mathrm{m} & \text { particle mass } \\ \mathrm{k} & \text { Boltzmann constant } \\ \mathrm{n}_{\mathrm{e}} & \text { electron density } \\ \mathrm{n}_{\mathrm{ref}} & \text { reference plasma density } \\ \mathrm{T} & \text { temperature, } \mathrm{K} \\ \mathrm{T}_{\mathrm{e}} & \text { electron temperature, } \mathrm{K} \\ \mathrm{T}_{\mathrm{i}} & \text { ion temperature, } \mathrm{K} \\ \Gamma & \text { particle flux } \\ \eta_{\mathrm{m}} & \text { propellant mass utilization } \\ \phi & \text { local plasma potential } \\ \phi_{0} & \text { bulk plasma potential in the discharge } \\ \sigma & \text { reaction cross section } \\ \mathrm{i} & \text { species index number }\end{array}$

\section{Introduction}

For ion engines, erosion of the accelerator grid is a principal life-limiting process and a primary source of thruster-induced spacecraft contamination."

${ }^{3}$ The usual grid material, molybdenum, is sputtered into the ion engine plume. It deposits on neighboring surfaces upon contact, leaving a metallic coating that alters surface properties and may affect the performance of solar arrays, sensors, and thermal control materials.

The limitation of lifetime due to accel erosion exerts considerable influence on the choice of thruster type and rated thrust for spacecraft. The end of life is usually taken at the point of grid mechanical failure due to extensive loss of the webbing. This results in loss of beam current capability and the onset of electron backstreaming into the discharge chamber. The erosion of grid material may

Coptright $\odot 1999$ by American Institute of Aeronautics and Astronautics. Inc. 
also produce flakes which electrically short adjacent grids as may have temporarily occurred on the recent NSTAR mission, ${ }^{4}$ causing premature failure unless the short can be cleared. For the more than 60 ion engine endurance tests which have been reported, the majority of failure modes involved the accel grid. ${ }^{\prime}$ To partially mitigate grid erosion effects, significant effort has been expended in the development of grids using erosionresistant materials. ${ }^{6.7}$

Many studies have been devoted to the subject of accelerator grid wear (for recent examples, see Refs. 8 and 9). During the past decades of ion engine research and development, the impingement of charge exchange (CE) ions has been accepted as the cause of the erosion under normal operating conditions. It has been recognized that under special circumstances, as when the perveance limit is exceeded, the grids are poorly designed or grid apertures are mis-aligned, direct impingement of ions at the ion beam energy or above may occur. These energetic ions have a far higher sputter yield than charge exchange ions ${ }^{10}$ which, with a typical -200 or $-300 \mathrm{~V}$ on the accel, impinge at a relatively low energy:

The impingement of $\mathrm{CE}$ ions increases at high background pressure, necessitating the use of high pumping speeds during testing. Reduction of the accel grid erosion rate is also achieved by incorporating a negatively biased decel grid and operating the accel at a negative potential of low absolute value to limit sputtering yield. ${ }^{\prime \prime}$

Considerable evidence exists that impingement of charge exchange ions is not the only cause of accel grid erosion under normal operating conditions. Several measurements on the $\mathrm{T} 5$ ion thruster, which has been extensively studied have shown that the accel current and erosion rate is far from zero when the utilization of xenon propellant is extrapolated to $100 \%$.

One of these measurements consisted of directly monitoring the laser-induced-fluorescence signal from T5-plume molybdenum atoms as a function of $\eta_{m} \cdot{ }^{12} \mathrm{~A}$ second monitored the rate of mass gain, largely from molybdenum. at a quartz crystal microbalance (QCM) as a function of $\eta_{m}$. obtaining a similar curve. ${ }^{13}$ The rate of mass gain as a function of background pressure further demunstrated that the grid erosion was only slightly affected by charge exchange in the background gas. ${ }^{14}$ The accel current exhibited similar dependence as the mass gain whereas decel-current percentage variation was several times higher. All of these results taken together indicated that the impingement of $C E$ ions was not the dominant grid erosion mechanism at the higher $\eta_{m}$ operating points. However, design and fabrication flaws of the triple grid set used in these studies (baseline triple grid set or BTS), and the known presence of decel direct impingement presented a limitation to any attempt to elucidate a secondary erosion mechanism. The flight-quality grids used in the present study were needed to address the issue.

Several studies have compared the accel mass loss over an extended period of operation with its calculated loss. The comparisons, based on current collection and the sputter coefficient for an assumed impingement energy of charge exchange ions, have frequently obtained puzzling results. ${ }^{8.14}$

\section{Experimental}

Details of the facility have been described previously. ${ }^{2}$ The test chamber is $5.5 \mathrm{~m}$ in length and 2.4 $\mathrm{m}$ in diameter. Two custom reentrant cryopumps of combined $\approx 70,000 \mathrm{l} / \mathrm{s}$ pumping speed on xenon hang inside the chamber, suspended from flanges. During thruster operation at a nominal thrust level of $18 \mathrm{mN}$, the chamber pressure was maintained at about $1.5 \times 10^{-6}$ Torr.

The thruster was mounted on a fixed stand in a side chamber with the ion beam directed across the main chamber. The beam dump consisted of aluminum plates covered with carbon-carbon composite panels, without water cooling.

In the present study, three different extraction grid sets of flight-quality were installed on the TS ion thruster already characterized by past studies, ${ }^{2}$ to perform design validation. All grids were subjected to a wear test of approximately 50 hours in duration. One of the grid sets, GS005, was constructed according to a different design and will not be further discussed.

The thruster is diagrammed in Fig. 1. For this study the anode was biased at $1100 \mathrm{~V}$, and the screen grid was at about $1060 \mathrm{~V}$.

The screen and decel apertures were 2.15-2.18 $\mathrm{mm}$ in diameter, with hole pitch about $2.44 \mathrm{~mm}$. The accel aperture diameter was $1.75-1.78 \mathrm{~mm}$ with similar pitch. Grid thicknesses were $0.25 \mathrm{~mm}$ for screen and decel, and $1.00 \mathrm{~mm}$ for the accel which was spaced $0.75 \mathrm{~mm}$ from the screen and $0.50 \mathrm{~mm}$ from the decel.

Grid set (GS) 003 had been previously operated for a period of roughly 50 hours at thrust levels $\leq 18$ $\mathrm{mN}$. There were no prior hours of operation on the other grid sets. The performance of the three grid sets varied substantially, with 003 showing the best behavior in general. This can be attributed to its prior period of operation, since grid sets 003 and 004 were otherwise virtually identical.

The critical parameter of propellant utilization efficiency, $\eta_{m}$, was determined from calibrations of flow shunted from the engine, monitoring rate of 
pressure rise in a known volume. No correction for multiply charged ions was made for the values listed in Table 1. Neutralizer flow was not included in the calculation of $\eta_{\mathrm{m}}$. It was assumed that the flow rates measured with engine off are identical to those obtained with engine on.

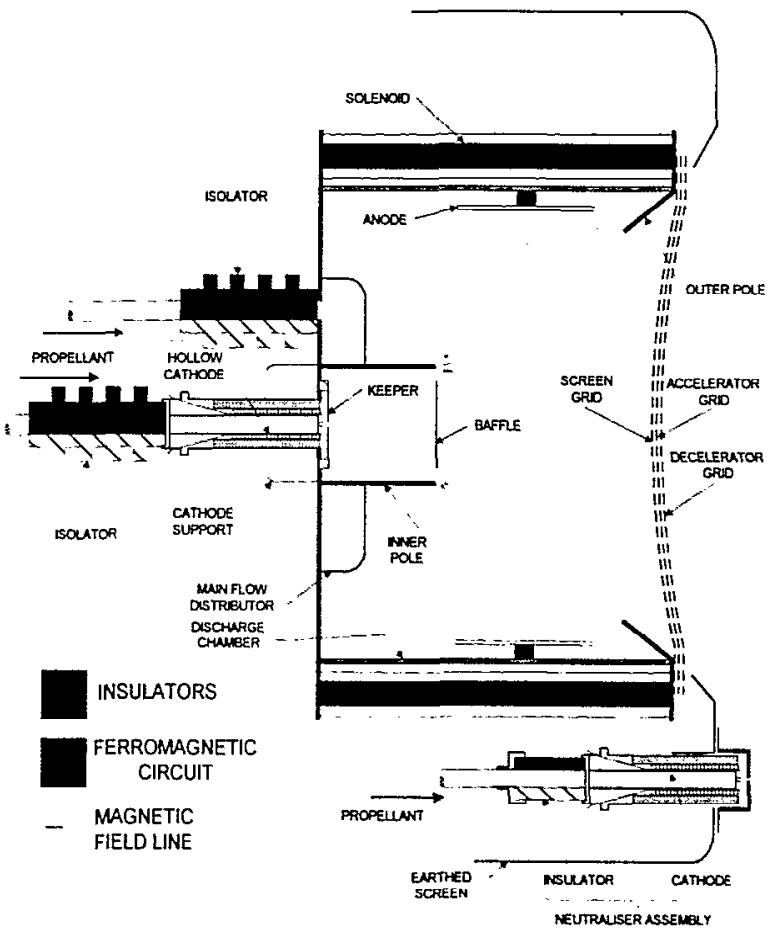

Figure 1. Schematic diagram of the T5 ion thruster.

\section{Experimental Results}

\section{Performance of grid set 003}

The observed thruster performance at several operating points is shown in Table 1. Previous performance figures obtained on the triple grid set (baseline triple grid set or BTS) used during the extensive prior characterization of the $\mathrm{T} 5$, are given in the table for comparison. Accelerator grid currents at the nominal $18 \mathrm{mN}$ thrust level are similar in the two cases. although it appears to be about 10\% higher for GS003 at $18 \mathrm{mN}$ and mass utilization near $85 \%$. Decelerator grid current level was about $45 \%$ lower for GS003 than for the BTS grid for the same $18 \mathrm{mN}$ operating points. The deposition rate measured by a QCM indicated that GS003 eroded much less rapidly than BTS: GS003 deposition rate was about $50 \%$ less so that grid erosion rate would be less by at least that amount. $^{1:}$ Since the accel current of the two grid sets was similar, it appears that the reduced deposition rate could be explained by a reduction in GS003 decel-grid erosion rate. The presence of direct impingement on the BTS decel had been documented previously. ${ }^{13}$ The major differences between the grid sets were (i) decel grid thickness of $0.25 \mathrm{~mm}$ for GS003 vs. $0.75 \mathrm{~mm}$ for BTS, and (ii) better aperture alignment and more uniform grid spacing for GS003. Both factors are qualitatively consistent with a substantial reduction in direct impingement by ions with energies determined by the full-voltage difference between screen and decel grids.

The decel current for the GS003 operating points of Table 1, when plotted against the normalized product of $\mathrm{I}_{\text {beam }}$ and $\left(1-\eta_{m}\right) / \eta_{m}$, follows a linear relation as shown in Fig. 2. The intersection of the linear decel fit at the $y$ axis would have the small, positive value of $0.1 \mathrm{~mA}$ if the uncorrected utilization were used. Instead, $\eta_{m}$ was corrected by a factor of 0.94 across the board, corresponding to about $12 \% \mathrm{Xe}^{+2}$ at each operating point. These results indicate that the decel current is dominated by the impingement of charge exchange ions. The accel current, in contrast, is not a wellbehaved function. In addition to a large amount of scatter, it appears to have a nonlinear relationship with the quantity $\left(1-\eta_{\mathrm{m}}\right) I_{\text {beam }} / \eta_{\mathrm{m}}$. A close examination of the figure reveals that the scattering pattern of accel and decel current is similar, but the decel deviations are an order of magnitude smaller.

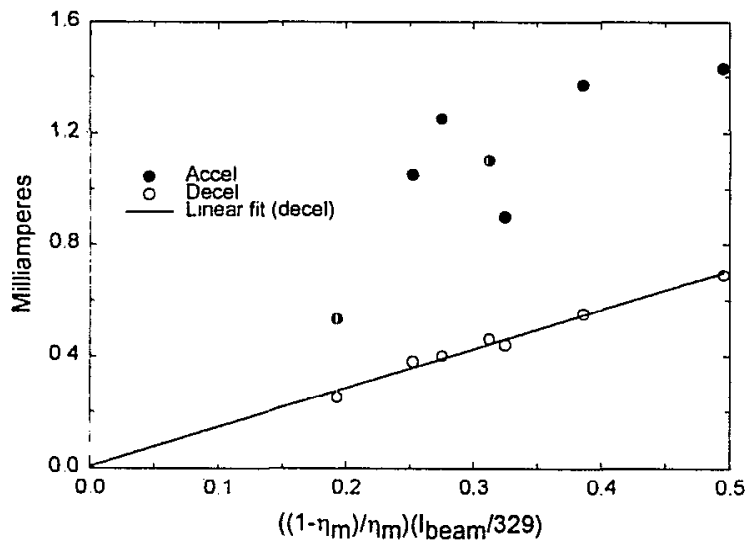

Figure 2. Plots of accel and decel current for GS003.

Charge exchange ions formed in the intergrid region are much more likely to impinge on the accel grid than the decel because the accel grid potential is normally $175 \mathrm{~V}$ more negative. $\mathrm{CE}$ ions formed downstream from the decel may strike its downstream face, but produce little erosion when they do so. It may 
be expected that $\mathrm{CE}$ ions which form downstream of the decel and migrate upstream have a relative probability of striking decel and accel grids that depends upon the open area fraction (OAF) of the decel grid. The potentials of the grids are determining factors as well.

Suppose that the rate at which $l_{\text {acc }}$ changes at fixed $I_{\text {beam }}$ due to the charge exchange ions formed downstream can be approximated by $\Delta \mathrm{I}_{\text {dec }}$ times (1-
OAF)/OAF, or $0.4 \Delta \mathrm{I}_{\text {dec }}$. The sum of impinging CE ions originating from downstream and intergrid regions is $\propto$ $\left(1-\eta_{\mathrm{m}}\right) / \eta_{\mathrm{m}}$. A comparison of operating points (1) and (6) of Table 1 then suggests that the accel grid collects roughly three times as many $\mathrm{CE}$ ions from the intergrid region as from downstream of the decel. Figure 6 of Ref. 13 suggests a larger factor of about 6 , measured with BTS.

Table 1. Operating parameters for selected operating points, grid set 003.

\begin{tabular}{lcccccccccc}
\hline \multicolumn{1}{c}{$\begin{array}{l}\text { OPERATING } \\
\text { POINT/CASE }\end{array}$} & $\mathrm{I}_{\text {beam }}$ & $\mathrm{V}_{\text {beam }}$ & $\mathrm{V}_{\text {anode }}$ & $\mathrm{I}_{\text {anode }}$ & $\mathrm{V}_{\text {acc }}$ & $\mathrm{I}_{\text {acc }}$ & $\mathrm{l}_{\text {dec }}$ & $\mathrm{V}_{\text {dec }}$ & $\mathrm{I}_{\text {acc }}+\mathrm{I}_{\text {dec }}$ & $\eta_{\mathrm{m}}{ }^{\mathrm{A}}$ \\
\hline (1) $17.9 \mathrm{mN}$ & 328 & 1100 & 40.7 & 2.50 & -225 & 1.10 & 0.46 & -50 & 1.56 & 81.0 \\
(2) 19.0 & 348 & $"$ & 43.0 & - & $"$ & 1.05 & 0.38 & $"$ & 1.43 & 85.9 \\
(3) 14.8 & 270 & $"$ & 40.8 & 1.85 & $"$ & 0.90 & 0.44 & $"$ & 1.34 & 76.2 \\
(4) 21.8 & 399 & $"$ & 43.0 & 2.90 & $"$ & 1.25 & 0.40 & $"$ & 1.65 & 86.7 \\
$(5) 19.9$ & 363 & $"$ & 38.9 & 2.90 & $"$ & 1.37 & 0.55 & $"$ & 1.92 & 78.8 \\
(6) 17.9 & 327 & $"$ & 37.2 & 2.90 & $"$ & 1.43 & 0.69 & $"$ & 2.12 & 71.0 \\
(7) 11.9 & 218 & $"$ & 44.8 & 1.50 & $"$ & 0.53 & 0.25 & $"$ & 0.78 & 82.4 \\
(8) 18.0 (BTS) & 329 & $"$ & 43.0 & 2.25 & $"$ & 0.90 & 0.65 & & 1.55 & 85.6 \\
\hline \hline
\end{tabular}

Apropellant utilization efficiency (\%). This figure relies on calibrations of flow shunted from the engine according to rate of pressure rise in a known volume.

Using the expression $\Delta \mathrm{I}_{\mathrm{acc}}^{\mathrm{E}} \cong 1.5 \Delta \mathrm{I}_{\text {dec }}$ and the assumption that decel grid current derives entirely from charge exchange ions, an estimate was obtained for the amount of $C E$ current, $I_{\text {acc }}^{\mathrm{E}}$, collected by the accel grid. The operating points of Table 1 were extrapolated to $100^{\circ}$ o utilization, and an estimate for the remaining accel current produced by processes independent of charge exchange (PIE) was obtained after subtracting the calculated CE current from the total. The result is plotted in Fig. 3 vs. beam current. It is obvious that fitting the PIE accel-grid current estimates to an equation does not require a

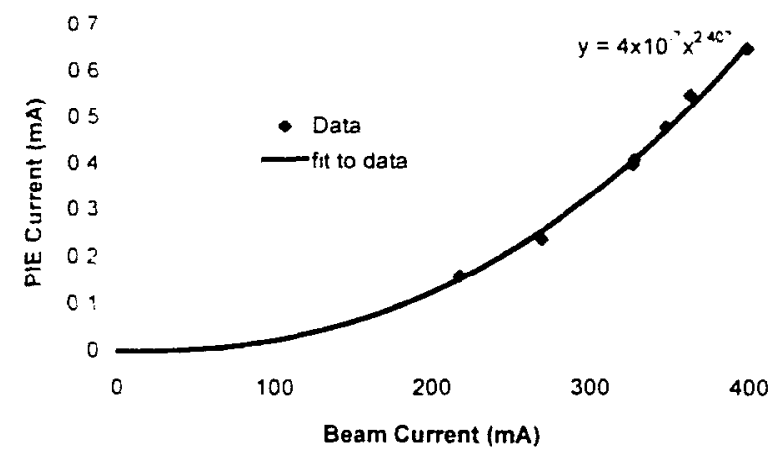

Figure 3. The estimated portion of GS003 accelerator current which is independent of charge exchange. as a function of beam current (sece lext) nonzero intercept, consistent with expectations. The estimated PIE current varies as $\mathrm{aI}^{\mathrm{b}}$, where $\mathrm{b}$ has a value of about 2.4 .

The ratio of accel to decel current level increases significantly with $I_{\text {beam }}$. Since it has already been established that the decel current is dominated by charge exchange ions, this provides further evidence for a sizable PIE contribution to the accel current. If charge exchange dominated the accel current the ratio would be constant.

One possible origin of PIE ions below the perveance limit was considered to be the Coulomb scattering of the ions. Impingement current due to such a process would depend on the square of beam current. Coulomb and ion-neutral scattering were therefore included in the modeling effort.

A significant number of the $C E$ ions striking the accel grid will do so at an energy substantially exceeding 225 $\mathrm{eV}$. This is because the charge exchange probability decreases with $\mathrm{Xe}^{*}$ energy, so that charge exchange is more likely per unit time during the acceleration phase than the extraction phase. For example, a CE ion formed when a xenon ion has traversed $50 \%$ of the screen-accel gap can impinge on the accel grid with an energy of about $0.50(1100-(-225))=668 \mathrm{eV}$. As a result, for a properly designed, properly operated extraction grid set with accel/decel voltages of $-225 /$ 50 , accel erosion rate is expected to dominate over decel erosion rate. 
Table 2. Operating parameters for selected operating points, grid set 004.

\begin{tabular}{ccccccccccc}
\hline \hline OPERATING POINT & $\mathrm{I}_{\text {beam }}$ & $\mathrm{V}_{\text {beam }}$ & $\mathrm{V}_{\text {anode }}$ & $\mathrm{I}_{\text {anode }}$ & $\mathrm{V}_{\text {acc }}$ & $\mathrm{I}_{\text {acc }}$ & $\mathrm{I}_{\text {dec }}$ & $\mathrm{V}_{\text {dec }}$ & $\mathrm{I}_{\text {acc }}+\mathrm{I}_{\text {dec }}$ & $\eta_{\mathrm{m}}$ \\
\hline$(1)$ & $\mathbf{3 9 8}$ & 1100 & 44.2 & 2.65 & -225 & 1.27 & 0.36 & -50 & 1.63 & - \\
$(2)$ & 457 & $"$ & 42.0 & $"$ & $"$ & 2.50 & 1.18 & $"$ & 3.68 & 80.3 \\
$(3)^{\mathrm{A}}$ & 456 & $"$ & 42.2 & $"$ & $"$ & 3.27 & 1.61 & $"$ & 4.87 & 80.1 \\
$(4)$ & 458 & $"$ & 42.7 & $"$ & $\mathbf{- 1 7 5}$ & 4.81 & 2.20 & $"$ & 7.01 & 80.5 \\
$(5)$ & 458 & $"$ & 42.1 & $"$ & $\mathbf{- 2 5 0}$ & 2.24 & 0.92 & $"$ & 3.16 & 80.5 \\
$(6)$ & 457 & $"$ & 41.9 & $"$ & $\mathbf{- 2 2 5}$ & 2.47 & 1.13 & $"$ & 3.60 & 80.3 \\
$(7)$ & 457 & $"$ & 40.7 & $\mathbf{3 . 0 0}$ & $"$ & 2.20 & 0.80 & $"$ & 3.00 & 80.3 \\
$(8)$ & 457 & $"$ & 42.2 & $\mathbf{2 . 6 5}$ & $"$ & 2.64 & 1.13 & $"$ & 3.77 & 80.3 \\
BTS & $\mathbf{4 5 7}$ & $"$ & 43.5 & $\mathbf{3 . 0 0}$ & $\mathbf{- 2 2 5}$ & 1.20 & 0.90 & $"$ & 2.10 & $\mathbf{8 8 . 0}$ \\
\hline \hline
\end{tabular}

${ }^{A}$ Cathode current set to $1.00 \mathrm{~A}$ rather than normal $0.65 \mathrm{~A}$ setting.

\section{Performance of GS 004}

As stated above, GS004 and GS003 were fabricated according to the same design specifications, but GS003 had undergone a brief prior period of testing. GS004 was operated at $25 \mathrm{mN}$ (see Table 2) during the majority of the test period. Its initial performance at $22 \mathrm{mN}$ was similar to GS003, however both accel and decel grid- current levels were quite high at the $25 \mathrm{mN}$ operating point. The accel and decel currents were highly sensitive to adjustment of the accel voltage (see Table 2), indicating direct impingement on each grid by energetic ions. At a beam current of $457 \mathrm{~mA}$, a reduction in discharge current on the third day of operation resulted in a substantial rise in both accel and decel current levels (see operating points 7,8). The reduced discharge current is compensated by increases in magnetic field strength and discharge voltage. Increased magnetic field strength improves electron confinement efficiency while increased discharge voltage raises the Bohm velocity and electron energy. These effects help to compensate for a reduced ion production rate associated with decreased discharge current. The ratio $\mathrm{Xe}^{-2}: \mathrm{Xe}^{*}$ increases rapidly with discharge voltage. ${ }^{15}$

The effect of main cathode current on grid current levels is even more pronounced than discharge voltage. This accounts for the high grid currents of operating point 3. for which the cathode current was $1.00 \mathrm{Amp}$ rather than 0.65 . the normal setting. In conjunction with the higher cathode current setting, $\Delta \mathrm{V}$ between cathode and keeper increased about 1.3 Volts whereas $I_{d}$ increased only $0.4 \mathrm{~V}$. Given the energy dependence of emitted ions on cathode current level measured in other studies. ${ }^{10}$ the elevated grid currents of operating point 3 may result from an upward shift in cathode ion energy distribution and flux level. The cathode ion flux will increase rapidly with cathode current, ${ }^{17}$ and these ions by virtue of their high initial velocity and broad velocity distribution, assuming they arrive at the grids with the same initial properties, will have a greater probability of accel/decel grid impingement. Based on these considerations, the velocity distribution function for ions entering the extraction grids may be quite important in determining grid impingement currents. As far as we are aware, neither the width of this distribution nor the effects of ion-ion (Coulomb) and ion-neutral scattering have been considered in previous ion engine models.

Figure 4 shows the grid currents observed at the $25 \mathrm{mN}$ operating point during most of the GS004 wear test. The trend is clearly down, except for an upward spike at about 1500 minutes. This spike was associated with increased recycling activity.

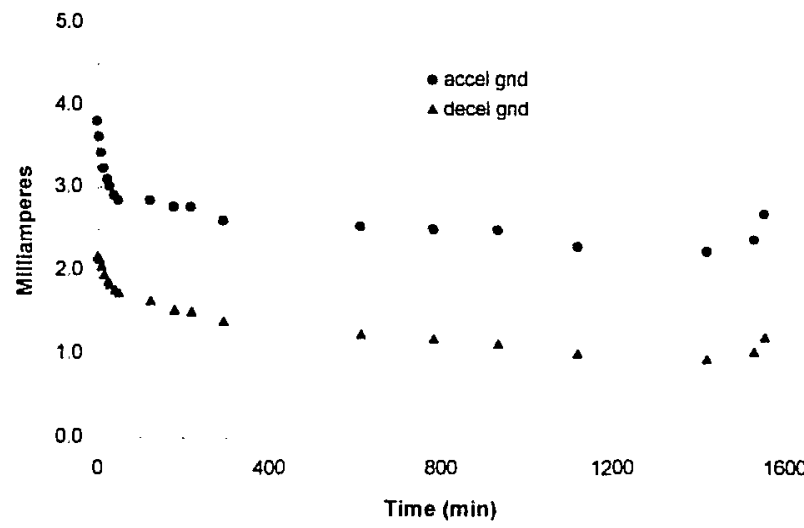

Figure 4. Plot of grid currents at the normal $25 \mathrm{mN}$ operating point (see Table 2) during the four days of GS004 wear testing.

\section{Modeling}

Computer models of flow through ion grid apertures have been developed previously by Arakawa and Ishihara ${ }^{18}$ and by Peng et al. ${ }^{19}$ In Ref. 18, a particle description of the ions was employed. Neutral flow was neglected as was charge exchange. In Ref. 19, the ions were again treated using particles but in this case 
charge exchange was included by assuming a constant neutral flow field. In both Refs. 18 and 19, only single charged ions were considered. In the present work, the ions and neutrals are treated in detail using a combination of two particle methods. The Particle-InCell method (PIC) ${ }^{20}$ is employed to model the plasma dynamics. In the present study, ions (both single and double charges) are treated as particles. Following the work of Peng et al ${ }^{19}$ electrons are only assumed to exist in the region upstream of the screen grid. A fluid approach is employed for the electrons in which their temperature, $T_{e}$, is assumed to be constant, and their number density, $\mathrm{n}_{\mathrm{e}}$, is given by the Boltzmann relation:

$$
n_{t^{\prime}}=n_{r e f} \exp \left(\frac{\phi-\phi_{0}}{T_{e}}\right)
$$

where $\phi$ is the local plasma potential, $\phi_{0}$ is the discharge potential $(1100 \mathrm{~V})$, and the electron temperature is in units of electron volts. The reference density, $\mathrm{n}_{\mathrm{ref}}$, is taken to be the total ion charge density employed in the upstream boundary conditions (see discussion below). The Poisson equation is solved on a rectangular grid where the ion density is given by the particles and the electron number density is given by the above equation. Following the approach of Roy et $\mathrm{al}^{21}$ an Alternating Direction Implicit (ADI) method is employed to solve the Poisson equation which is non-linear due to the presence of $\phi$ in the above equation for the electron number density.

The second particle technique employed is the direct simulation Monte Carlo method (DSMC) ${ }^{22}$ which is used to model collision phenomena. Thus, the neutral atoms are also treated as particles, and three different types of collisions are modeled: (1) momentum transfer using the cross sections of Dalgarno et al: ${ }^{23}$ (2) charge exchange between $\mathrm{Xe}$ and $\mathrm{Xe}^{*}$ using the cross sections of Sakabe and Izawa ${ }^{2-4}$ and between $\mathrm{Xe}$ and $\mathrm{Xe}^{* 2}$ using the following curve fit to the measurements of Hasted and Hussain:

$\sigma=\left(3.4069 \times 10^{-9}-2.7038 \times 10^{-10} \log (g)\right)^{2} m^{2}$

and (3) Coulomb collisions. For Coulomb collisions, the equations from classical plasma physics for cross section and scattering angle are employed, e.g. see Ref. 25. However, the scattering angle for a particular collision is sampled statistically from the distribution suggested by Nanbu." This procedure is necessary because DSMC-PIC is a statistical approach and the method does not compute intersecting particle trajectories deterministically. In Fig. 5, a selection of the collision cross sections employed in the present study is shown for the xenon system as a function of relative velocity. It can be concluded that Coulomb collisions dominate at low collision energies. At high energy, the charge exchange cross section dominates. In Fig. 6, the variation of Coulomb scattering angle with relative velocity is shown. Here, it is evident that only very glancing interactions occur even at the low relative velocities which provide the largest cross sections (again see Fig. 5).

The upstream boundary conditions for the DSMC-PIC approach involve specification of species number densities, velocities, and temperatures. The temperature of the neutral atoms is assumed to be 500 $\mathrm{K}$. The temperature of the ions is taken to be $4 \mathrm{eV}$ based on the measurements of Pollard. ${ }^{15}$ The electron temperature is not well known and a value of $5 \mathrm{eV}$ is assumed here. These temperatures are employed to calculate the atom and ion velocities of particles entering the flow domain from the discharge chamber. In each case, the flux of species i across a radial surface of area A upstream of the screen grid is given by the standard result from kinetic theory:

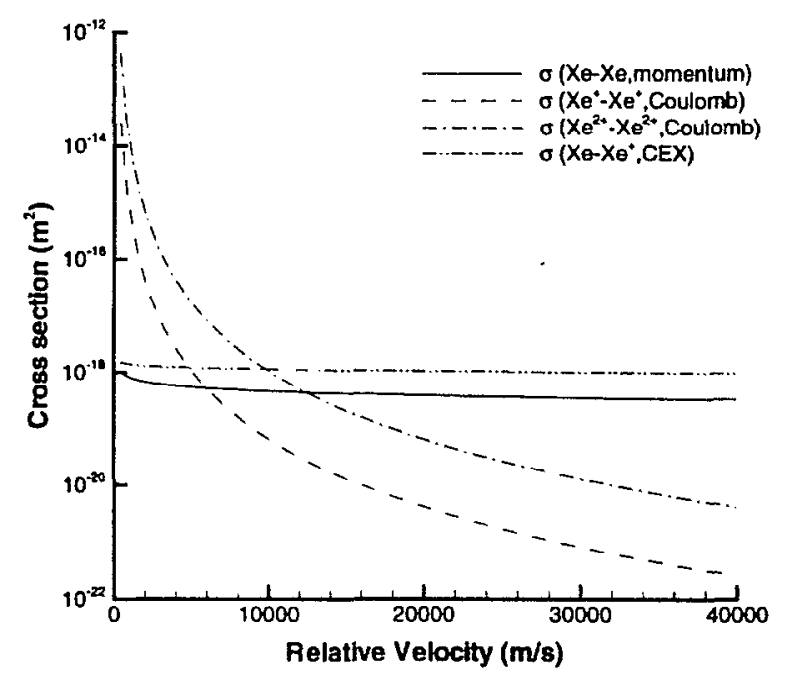

Figure 5. Collision cross sections as a function of relative velocity.

$$
\Gamma_{1}=\frac{1}{4} n_{1} C, A
$$

where the mean molecular speed is given by:

$$
C_{1}=\sqrt{\frac{8 k T_{1}}{\pi m_{1}}}
$$




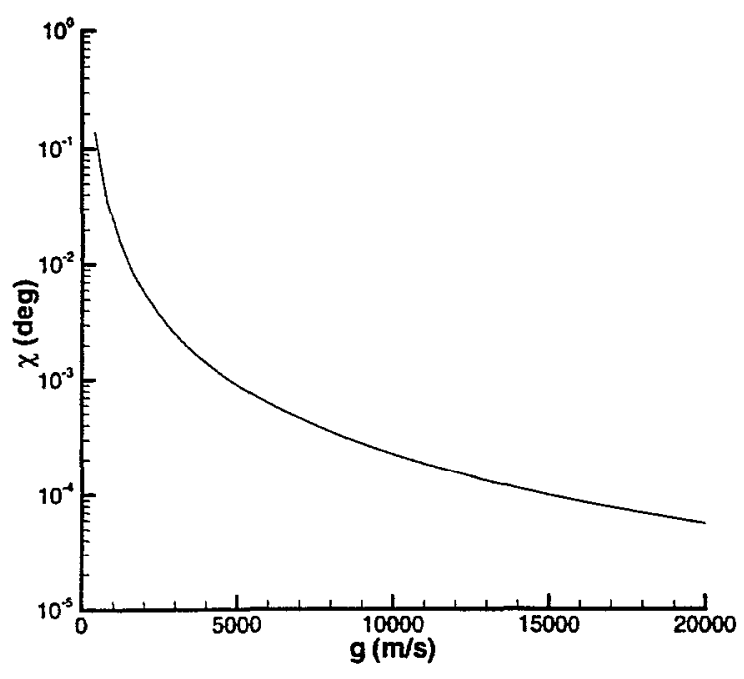

Figure 6. Coulomb collision scattering angle as a function of relative velocity.

The atom and ion fluxes are computed so as to be consistent with the experimentally measured values of total beam current and propellant efficiency. Thus, using Eq. (3), the atom and ion number densities are determined. One further parameter that cannot be determined from the measured systems parameters is the fraction of ions that are double charged. Based on the measurements of Pollard ${ }^{15}$ it is expected that this fraction lies between 0.05 and 0.20 . Various values are employed in the computations.

The computational grid employed in all simulations is shown in Fig. 7. The cell size is less than the smallest Debye length considered in these investigations. In all cases, the cell sizes are orders of magnitude smaller than the mean free paths for all flow conditions. The time-step employed in the simulations is the reciprocal of the plasma frequency. The surfaces of the three screens are at fixed potentials and are assumed to have a constant temperature of $500 \mathrm{~K}$. Ions striking any of the screens are assumed to recombine to neutral atoms. The facility back pressure is sufficiently low such that it has virtually no effect on the flow through the screens. It is therefore neglected in the computations.

The main purpose of the computations is to predict the ion current to the acceleration grid. This is computed by directly counting the number of ion particles impacting on any surface of the acceleration grid over a finite period of physical time. The current collected by the deceleration grid is not considered here

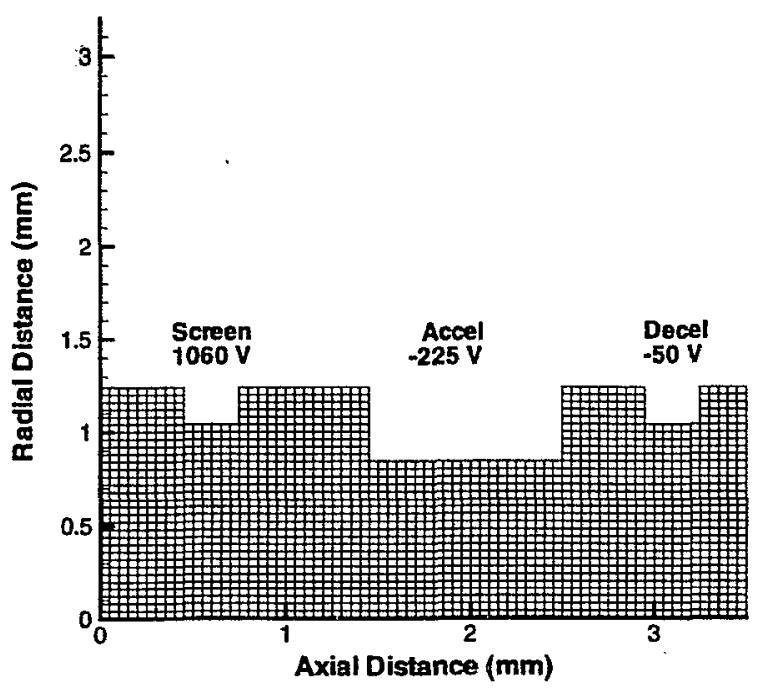

Figure 7. Computational grid employed.

since an adequate treatment of the neutralized plasma downstream of the thruster exit plane is required. This is left for future study.

\section{Computational Results}

Four operating points of GS003 catalogued in Table 1 , are investigated computationally, cases $3,4,6$, and 7.

For each of these cases, three simulations are computed in which the fraction of double charged ions is set to $0.0,0.1$, and 0.2 . In addition, further computations are performed for cases 4 and 6 to assess the sensitivity of the solutions to the value of the electron temperature assumed, and to test the effect of the different types of collisions.

In Fig. 8, contours of plasma potential are shown for a computation of Case 6 with $10 \%$ double charged ions. These have the expected shape seen in previous computational studies, ${ }^{1819}$ which result in the ions first being accelerated away from the screen grid, and finally pulled up towards the acceleration and deceleration grids.

In Fig. 9, the variation of current collected on the acceleration grid as a function of total beam current is shown. The computational results indicate a sensitivity to the fraction of double charged ions assumed in the discharge plasma. It is found that lower fractions of $\mathrm{Xe}^{-2}$ lead to smaller current being collected on the acceleration grid. Close examination of the computational results revealed the explanation for this phenomenon. When there is a significant fraction of $\mathrm{Xe}^{\cdot-2}$ in the plasma, the average ion velocity is increased 


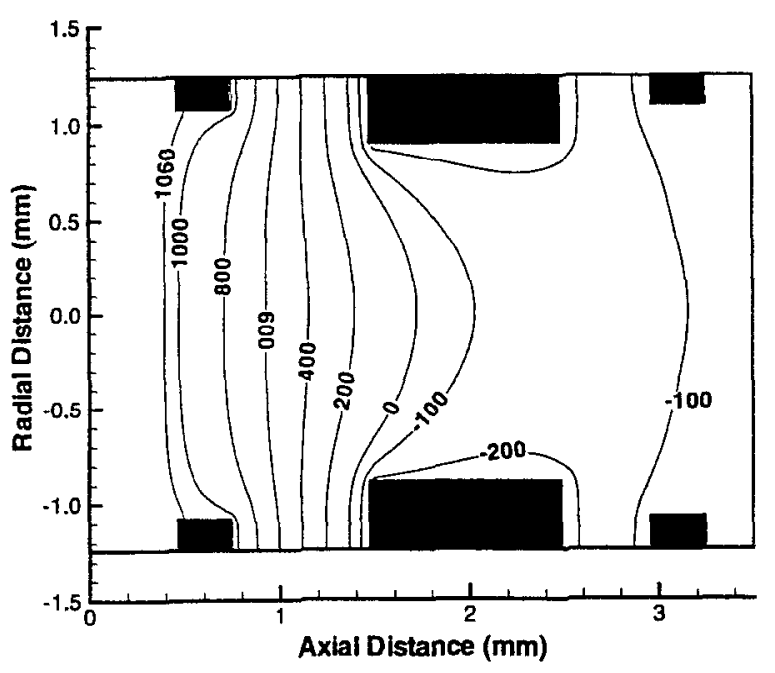

Figure 8. Contours of plasma potential for Case 6.

since the electric fields accelerate the double charged ions to higher velocities than the single charged ions. In terms of the current collected by the acceleration grid, this effect is most important in the region next to the screen grid where the ions are accelerated by the potential gradients in the direction towards the centerline of the aperture. Hence, in the case where there is a finite fraction of $\mathrm{Xe}^{+2}$, more of the plasma is accelerated towards the centerline in comparison to the case where there is no $\mathrm{Xe}^{* z}$.

Returning to the data shown in Fig. 9, in general, the computational results using $10 \%$ of ions having double charge offers good agreement with the experimental data at low beam current. At the highest beam current, it is necessary to increase the fraction of $\mathrm{Xe}^{\mathrm{r}}$ to $20^{\circ} \%$ to obtain agreement with the measured data. These values are consistent with the experimental measurements of multiple charged ion fractions as a function of thruster operation conditions reported by Pollard."

The computational results shown in Fig. 9 include all collision mechanisms and employ an electron temperature of $5 \mathrm{eV}$. Further computations indicated that omission of the charge exchange collisions leads to a reduction in the accel current by values lying between 15 and $45^{\circ}$ o depending on the specific conditions. Omission of the Coulomb collisions has no effect on the current collected. For operating point 6 , increasing the electron temperature to $10 \mathrm{eV}$ decreased the current collected b! $23^{\circ}$ o. For the same case, decreasing the electron temperature to $4 \mathrm{eV}$ increased the acceleration grid current by $13^{\circ}$. These results are explained by the fact that the potential in the Boltzmann equation is directly proportional to the electron temperature.

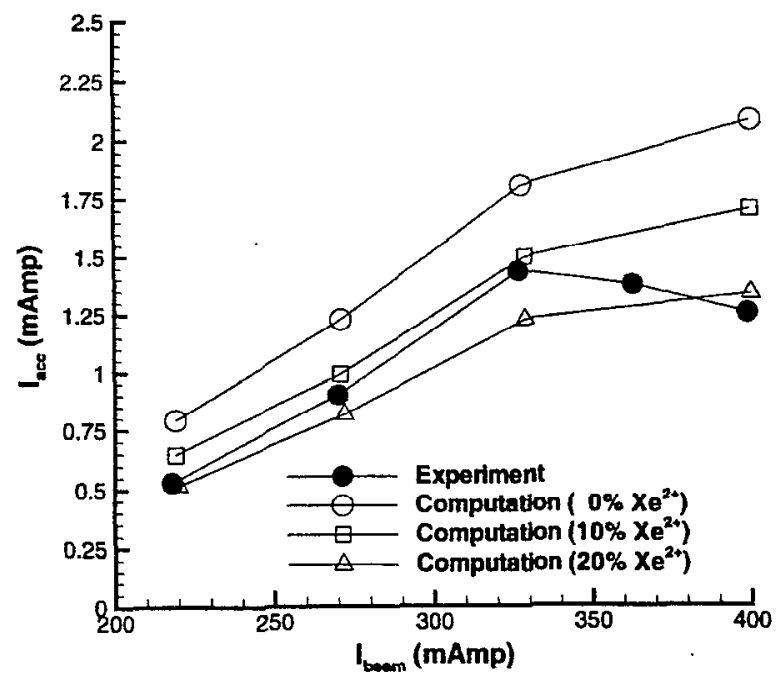

Figure 9. Variation of current collected on acceleration grid as a function of total beam current.

Hence, the spatial derivatives of potential (that is, the electric fields) are also proportional to the electron temperature. At higher electron temperature, there is a greater acceleration of ions away from the screen grid than is simulated at lower electron temperature. Thus, as the electron temperature is increased, there is a decrease in current collected on the acceleration grid.

Therefore, although the agreement obtained between experiment and computation as shown in Fig. 9 is good, there is some level of uncertainty in the simulation results particularly in terms of specifying the discharge plasma parameters of electron temperature and fraction of double charged ions.

\section{Discussion}

All of the previous modeling studies of ion engine plasma and ion extraction appear to have assumed ion temperature similar to neutral atom temperature rather than electron temperature. Similarly, ion temperature was not determined in the Langmuir probe study of the discharge chamber, and it was thought to be two orders of magnitude below $\mathrm{T}_{\mathrm{e}}{ }^{27}$

Recently, experimental and theoretical evidence has become available indicating that the ion temperature in low density plasmas can be very substantial. Numerical models of plasma jet propagation for the SPT-100 and SPT-140 Hall thrusters have utilized ion temperatures between 4 and $40 \mathrm{eV}$ to reach agreement with experiment. ${ }^{28.29}$ Some recent studies of plasma reactors and the ion etching process have found ion temperature to be far above the wall temperature. . $^{30.31}$ 
Due to the potential gradient in the discharge chamber and the need for high ion migration rates to the screen grid during thruster operation, most ions may be expected to have kinetic energy of $1-5 \mathrm{eV}$ at the time they encounter the sheath. While the T5 far-field measurement of ion energy distribution ${ }^{15}$ is consistent with an ion temperature of $4 \mathrm{eV}$, direct measurements of the ion velocity distribution and other properties of the discharge plasma would be valuable.

The PIE current may depend on various parameters of the discharge plasma, shape of the plasma sheath associated with individual screen grid apertures, geometry and extraction voltages of the extraction grid set. PIE ions will impinge at high energy and produce much more erosion and grid damage per ion than typical $\mathrm{CE}$ ions. Given this fact and the possible influence of PIE ions in previous studies of accel erosion, prior experimental and thcorctical work on accel wear may need to be reevaluated for all ion engines.

Decel current for GS003 is dominated by $\mathrm{CE}$ ions over the range of operating points in Table 1, whereas accel current also has a large contribution from PIE ions. PIE ion impingement will primarily erode accel barrels, whereas $C E$ ions may erode the grid face as well. The decel diameter is considerably larger than the accel diameter (see Fig. 7), which probably accounts for its observed lack of PIE ion impingement.

Both impingement and erosion rate can readily be dominated by $C E$ ions at low utilization, particularly when the accel grid operating voltage is at high absolute value. The effects of PIE ions are likely to dominate at high utilization, especially if a triple grid system is employed with a low (absolute) voltage on the accel.

\section{Concluding Remarks}

The $X e^{*}$ flux at ion beam energy is known to drop by roughly an order of magnitude per 10-deg angular rotation away from the thrust axis, in the far-field, with no sharp discontinuities. This behavior is qualitatively consistent with a radial ion temperature that is far higher than the discharge chamber wall, or the influence of various scattering processes. The latter effects were found to be minor in this study.

The collected grid currents are attributed to two main sources. one of which is determined by the details of charge exchange ion formation and migration, and the other by processes independent of charge exchange. Both sources are significant for the accelerator grid, whereas charge exchange ions dominate deceleratorgrid current.
The use of flight-model grids was essential. Previous T5 grid sets were inadequate for study of impingement current because of the inferior grid concentricity and direct impingement commonly collected by the decel grid. The performance of the flight-model grids was very good, with accel and decel impingement current as low as $0.24 \%$ and $0.10 \%$, respectively, of the beam current.

The erosion by PIE ions should be self-limiting to a degree, since the enlargement of accel apertures will tend to slow the erosion rate. This would explain the downward trend of collected accel current and recorded QCM mass gain over long periods of thruster operation.

This study purports to have discovered a new mechanism of extraction grid erosion in ion thrusters. The result is of considerable potential importance for the improvement of ion engine lifetime and reduction of contamination effects, since it may be possible to tailor the discharge chamber for plasma properties which allow the reduction of grid erosion. While the agreement between theory and experiment is better here than for previous modeling studies, a number of uncertainties remain to be resolved. Further measurements will be needed to obtain detailed information on the plasma properties in the ion engine discharge chamber and at the entrance apertures of the screen grid.

\section{Acknowledgments}

The preparation of this manuscript was supported in part by The Aerospace Corporation through the Aerospace Independent Research and Development Program. The authors wish to thank the Defence Evaluation and Research Agency for providing the grid sets and N. Wallace, D. Mundy, P. Wheldon, and H. Simpson for their efforts during the data collection phase of April-May 1996 at Aerospace, also D.G. Fearn and J.E. Pollard for useful discussions. Figure 1 was provided by $D$. Mundy.

\section{References}

1. Brophy, J.R.. Polk. J.E.. and Rawlin, V.K., "Ion Engine Service Life Validation by Analysis and Testing," AIAA Paper 96-2715, 32nd Joint Propulsion Conf., July 1996, and references therein.

2. Crofton, M.W.. "Evaluation of the United Kingdom Ion Thruster." J. Spacecraft and Rockets, Vol. 33, No. 5, 1996, pp. 739-747, and references therein.

3. Fearn. D.G., "Ion Thrusters Lifetime Limitations Imposed by Sputtering Processes," IEPC Paper 93-177,

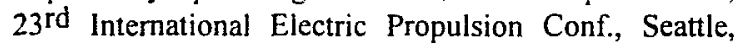
Sept. 1993. 
4. Press release 98-60, NASA Glenn Research Center, http://www.grc.nasa.gov/www/PA0/pressrel/98_60.htm.

5. Samanta Roy, R. I., Hastings, D. E., and Gatsonis, N. A., "Ion-Thruster Plume Modeling for Backflow Contamination," J. Spacecraft and Rockets, Vol. 33, 1996, pp. 524-534.

6. Kitamura. S. Hayakawa, Y., Kasai, Y.. and Ozaki, T., "Fabrication of Carbon-Carbon Composite Ion Thruster Grids - Improvement of Structural Strength," IEPC Paper 97-093, 25th International Electric Propulsion Conf., Cleveland. Aug. 1997: and references therein.

7. Shimada, S.. et al, "Ion Thruster Endurance Test using Developmental Model Thruster for ETS-VI." IEPC Paper 93-169. 23rd International Electric Propulsion Conf.. Seattle. Sept. 1993.

8. Polk. J.E.. Anderson. J.R.. Rawlin. V.K., Patterson, M.J., and Sovey. J., "In Situ, Time-Resolved Accelerator Grid Erosion Measurements in the NSTAR 8000 Hour Ion Engine Wear Test." IEPC Paper 97-047. 25th International Electric Propulsion Conf., Cleveland, Aug. 1997.

9. Tartz. M.. Hartmann, E.. Deltschew, R., and Neumann. H.. "Grid Erosion Study of a Three-Grid Ion Thruster," AIAA Paper 98-3646. 34th Joint Propulsion Conf., Cleveland. July 1998.

10. Shutthanandan. V.. et al. "On the Measurement of LowEnerg: Sputtering Yield using Rutherford Backscattering Spectrometry," IEPC Paper 97-069. 25th International Electric Propulsion Conf., Cleveland. Aug. 1997

11. Brophı, J.R.. Pless, L.C., and Garner. C.E., "Ion Engine Endurance Testing at High Background Pressures." AIAA Paper 92-3205. 28 $8^{\text {th }}$ Joint Propulsion Conf. Nashville. Jul! 1992.

12 Crofton. MU.. "Laser Spectroscopic Study of the T5 Ion Thruster." AIAA Paper 95-2921, 315t Joint Propulsion Conf.. San Diego. July 1995.

13 Ahmed. L.N.. and Crofton, M. W... "Surface Modification Measurements in the T5 lon Thruster Plume." J. Propulsion and Power. Vol. 14. No. 3. 1998. pp. 336$34^{-}$

It Brophy, I.R. Carner. C.E. Gondfellow, K.D. Pivirotto. I J. and Polk. J.E.. "Electric Propulsion System Technologs." Annual Report-1991. No. 92-10. Jet Propulsion 1.aboratory. Pasadena. CA. Nov. 1992.

15 Pollard. J.1.. "Plume Angular. Energy, and Mass spectral Measurements with the Ts Ion Engine." AlAA Paper 95-2920. 31 st Joint Propulsion Conf., San Diego. Juls 1995

16. Predh. V.J. and Wilbur. P.J.. "High Current Hollow (athode Phenomena." I Propulsion and Power. Vol. 8. so 3. 1992. pp 635-6+3

1? Crofton. $11 \mathrm{~W}$. *A Small Diagnostics Facility for Electric Propulsion Issues: Initial Hollow Cathode Results." MAA Paper 99-(0454. 37th Aerospace Scuences Mertung. Jan 1999
18. Arakawa, Y. and Ishihara, K., "A Numerical Code for Cusped Ion Thrusters," IEPC Paper 91-118, October 1991.

19. Peng, X., Keefer, D., and Ruyten, W., "Plasma Particle Simulation of Electro-static Ion Thrusters," $J$. Propulsion and Power, Vol. 8, 1992, pp. 361-366.

20. Birdsall, C. K. and Langdon, A. B., Plasma Physics Via Computer Simulation, Adam Hilger Press, 1991.

21. Samanta Roy, R. 1., Hastings, D. E., and Gatsonis, N. A., "Ion-Thruster Plume Modeling for Backflow Contamination," J. Spacecraft and Rockets. Vol. 33, 1996, pp. 524-534.

22. Bird, G. A., Molecular Gas Dynamics and the Direct Simulation of Gas Flows, Oxford University Press, 1994.

23. Dalgarno, A., McDowell, M. R. C., and Williams, A., "The Mobilities of Ions in Unlike Gases." Proceedings of the Royal Society, Vol. 250, 1958, pp. 411-425.

24. Sakabe, S. and lzawa, Y., "Simple Formula for the Cross Sections of Resonant Charge Transfer Between Atoms and Their lons at low Impact Velocity." Physical Review $A$, Vol. 45, 1992, pp. 2086-2089.

25. Hasted, J. B. and Hussain, M., "Electron Capture by Multiply Charged lons," Proceedings of the Physical Society, London. Vol. 83, 1964, pp. 911-924.

26. Nanbu, K., "Theory of Cumulative Small-Angle Collisions in Plasmas," Physical Review E, Vol, 55, 1997, pp. 4642-4652.

27. Masek, T.D.. "Plasma Properties and Performance of Mercury Ion Thrusters", AlAA Journal, Vol. 9. No. 2, 1971, pp. 205-212.

28. Boyd. I.D." "Computation of the Plume of the D55 Hall Thruster." AIAA Paper 98-3798, 34th Joint Propulsion Conf., Cleveland. July 1998.

29. Bishaev. A.M., Kalashnikov, V.K., Kim. V., and Shavykina. A.V., "Numerical Modeling of the Propagation of a Plasma Jet Produced by a Stationary Plasma Thruster in a Low-Prcssurc Gas," Plasma Physics Reports, Vol. 24. No. 11, 1998, pp. 923-928.

30. Lymberopoulos. D.P.. Wise. R.S.. Economou. D.J., and Bartel. T.J.. "Ion Density and Temperature Distributions in an Inductively Coupled High-Plasma Density Reactor." IEEE Transactions on Plasma Science, Vol. 24. No. 1. 1996. pp. 129-130.

31. Zheng. J., Brinkmann. R.P., and McVittie. J.P., "The Effect of the Presheath on the Ion Angular Distribution at the Wafer Surface." J. Vacuum Science Technol. A. Vol. 13. No. 3. 1995. pp. 859-864 